\title{
Del conocimiento moral común de la razón al conocimiento filosófico
}

\author{
From common moral knowledge of reason to philosophical knowledge
}

\author{
Ana María Fajardo Fajardo ${ }^{1}$ \\ Universidad Externado de Colombia
}

Recibido: 15.11 .2020

Aceptado: 29.11.2020

\section{Resumen}

El libro de Inmanuel Kant titulado "Fundamentación de la metafísica de las costumbres", publicado en el año de 1785, es un trabajo sobre la filosofía de la moral; consta de un prefacio y tres capítulos. Cada capítulo nos lleva por un camino de ascenso: se trata, como dice el mismo autor, de un tránsito. Comienza analizando las convicciones éticas más comunes, para hablarnos luego de un ascenso que consiste en ir del plano común al filosófico, y de éste al metafísico, lugar donde se asienta la moral. Después de determinar el lugar donde se fundamenta la moral, nos dice Kant, se ha de descender nuevamente al plano común, es decir, que se ha de señalar cómo la razón pura puede hacerse práctica, ya que conviene fundamentar primero la moral en la razón pura, y luego sí, a partir de su fundamentación, enjuiciar las acciones morales.

En este artículo reflexionaremos sobre el capítulo primero que trata del tránsito del conocimiento moral común de la razón al conocimiento filosófico.

\footnotetext{
1 anafajardo03@est.uexternado.edu.co https://orcid.org/0000-0001-8103-8382
}

Volumen 1. Número 2. Julio - Diciembre 2021 
Palabras clave: Ciencias de la Educación, Filosofía, Ética, Lógica, Naturaleza humana, Ontología, Existencialismo.

\begin{abstract}
Inmanuel Kant's book entitled "Foundation of the metaphysics of customs", published in the year 1785, is a work on the philosophy of morality; It consists of a preface and three chapters: Each chapter takes us on a path of ascent: it is, as the same author says, a transit. It begins by analyzing the most common ethical convictions, and from there talk about an ascent that consists of going from the common to the philosophical plane, and from this to the metaphysical, place where morality is based. After determining the place where morality is based, Kant tells us, one has to descend again to the common plane, that is, it must be pointed out how pure reason can be made practical, since it is convenient to base morality first on reason pure, and then yes, from its foundation, to judge moral actions.
\end{abstract}

In this article we will reflect on the first chapter that deals with the transition from common moral knowledge from reason to philosophical knowledge.

Keywords: Education Sciences, Philosophy, Ethics, Logic, Human nature, Ontology, Existentialism.

\title{
1. Tránsito del conocimiento moral común de la razón al conocimiento filosófico
}

El libro titulado "fundamentación de la metafísica de las costumbres" su primer capítulo lo titula "del conocimiento moral popular al filosófico"; el siguiente, "de la filosofía moral popular a la metafísica de las costumbres" y, finalmente, “de la metafísica de las costumbres a la crítica de la razón pura práctica”. En este capítulo final, Kant enfatizará en la pregunta de ¿cómo puede ser posible el imperativo categórico? En este apartado nos dirá que esto es posible sólo a partir de la libertad (Avila Martinez, 2014). Para demostrar este hecho, el autor propone la dualidad entre mundo sensible y mundo inteligible. En el primero, el hombre como 
miembro del primer mundo participa de la causalidad y como ser perteneciente al mundo inteligible se puede pensar como un ser libre.

En el prefacio, el autor muestra una mínima clasificación del saber filosófico, el cual está dividido, según él, en filosofía formal y material. La primera es la lógica en tanto que cumple con la característica de la universalidad y la necesidad; la segunda se refiere a la física y a la moral; esta última -la moral- está constituida a partir de elementos empíricos y racionales, es decir, desde presupuestos de la experiencia y la metafísica; lo cual constituye un error para Kant, ya que, según él, debe hacerse una distinción entre lo empírico y lo metafísico (Plata Pineda, 2011). Nuestro filósofo se propone fundar una moral que esté alejada en sus fundamentos de todo lo que sea empírico.

Este contraste, de lo contingente y lo metafísico, le permite mostrar cómo la filosofía moral que él propone evita toda mezcla de lo empírico y lo metafísico, y se asienta en principios puros de la razón (Carvalho, 2015), formando así los conceptos básicos que constituyen su postura moral, tales como: a priori, ley, universalidad, objetividad, deber, autonomía, buena voluntad, libertad, etc. El motivo de tener claridad en la clasificación de la ciencia es que se puede llegar a saber con suma precisión hasta dónde puede construir la razón pura sus conceptos y de qué fuentes toma esa enseñanza a priori. En palabras de Kant:

He de limitarme a preguntar aquí si la naturaleza misma de la ciencia no exige que se separen siempre con gran cuidado la parte empírica y la parte racional; para poder saber cuánto puede construir la pura razón en ambos casos y de qué fuentes toma esa enseñanza a priori racionalidad. (Kant, 1985, p. 45)

Después de hacer la clasificación de las ciencias, se pasa a argumentar la necesidad de una moral racional pura, a priori, asunto éste que ya hemos tratado anteriormente, concretamente en el apartado que se titula (característica fundamental de la obra). La necesidad de una moral pura a priori está estrechamente relacionada con la idea que tiene Kant del deber y de las leyes (Lafarga \& Pegenaute, 2016); para nuestro filósofo, una ley ha de cumplir la característica de 
ser universal y necesaria, es decir objetiva; si no cumple con esta condición, no puede constituirse como ley moral. La razón de ser de la objetividad de la ley radica precisamente en el hecho de que ninguna persona se vea libre del cumplimiento de su deber. Según Kant, las leyes morales se caracterizan por estar libres de lo empírico (González, 2019). La moral kantiana, al constituirse como universal y necesaria, tiene que ir a parar inevitablemente a conceptos puros, a priori, es decir, a una metafísica de las costumbres (del Luján Di Sanza, 2014).

\section{Tránsito del conocimiento moral común de la razón al conocimiento filosófico}

Pasos del primer capítulo: la buena voluntad, la razón como rectora de la voluntad, el deber como núcleo fundamental de la voluntad, las características del deber (acción realizada conforme al deber y por deber), el valor moral de la acción, la formulación de la ley, y finalmente la necesidad de pasar del conocimiento moral ordinario al filosófico.

Kant inicia su argumentación con una afirmación enfática, sorprendente; nos dice al comienzo de este primer capítulo que no hay nada incondicionalmente bueno, excepto una buena voluntad:

$\mathrm{Ni}$ en el mundo, ni, en general, fuera de él es posible pensar nada que pueda ser considerado bueno sin restricción excepto una buena voluntad... La buena voluntad no es buena por lo que efectúe o realice, ni por su aptitud para alcanzar algún determinado fin propuesto previamente, sino que sólo es buena por el querer, es decir, en sí misma, y considerada por sí misma es sin comparación muchísimo más valiosa que todo lo que por medio de ella pudiéramos realizar en provecho de alguna inclinación. (Kant, 1985, p. 53)

Parece ser que antes de la formulación ya citada, Kant se preguntase: ¿qué es lo que hace que las cualidades y ciertas facultades que posee el ser humano sean buenas? A este interrogante

Volumen 1. Número 2. Julio - Diciembre 2021 
responde nuestro filósofo: lo que hace que todas estas disposiciones - cualidades y facultadessean buenas, es la buena voluntad (Iracheta Fernández, 2009).

El entendimiento, el ingenio, la facultad de discernir, el valor, la decisión, la constancia, el poder, la riqueza, la salud, la satisfacción, la alegría, el honor, en fin, la felicidad; por otra parte, el dominio de sí mismo, la moderación en afectos y pasiones, la sobria reflexión..., todas estas cualidades y facultades son buenas gracias a la voluntad que las hace buenas; sin ella podrían todas estas cualidades y facultades ser sumamente malas. En suma, lo único bueno es la voluntad considerada en sí misma (Aparicio-Gómez, 2006). Pero ¿qué significa eso de que la voluntad es buena sin restricción? Por un lado, esto quiere decir que la buena voluntad, como la propone Kant, es un postulado de la razón -es una voluntad pura, objetiva; el filósofo de Königsberg no se refiere a la voluntad de éste o aquel individuo, sino a la toma en sentido universal; es una representación ideal de la razón en la cual han de coincidir todas las voluntades individuales (Gallo, 2015).

Efectivamente, nos dice Kant, la voluntad es buena sin restricción, en sí misma. La buena voluntad no es buena por lo que realice, o por el fin que se pretenda alcanzar. Ella brilla como una joya, pues ni la utilidad ni la esterilidad pueden añadir o quitar nada a este valor. En cambio, las cualidades y facultades mencionadas anteriormente son buenas sólo en la medida en que cumplan con su objetivo; la felicidad por ejemplo será buena si es recompensa de la virtud.

A este querer han de apuntar las acciones morales; según la visión de Kant, la buena voluntad tiene un valor absoluto, independiente del fin y de las consecuencias de sus propósitos; es una facultad que no está condicionada por nada (Martínez Martínez, 2017). Esta clarificación de la voluntad como buena en sí misma, viene tras el análisis del concepto de deber.

Enseguida de la precisión anterior, Kant pasa a considerar la finalidad de la razón; nos dice que todos los hombres aspiran a la felicidad, y que la razón no es la más adecuada para proporcionarnos este objetivo, ya que ella no puede determinar objetivamente la felicidad, 
porque cada cual tiene una concepción distinta de la misma. Para lograr este objetivo nos bastaría simplemente con seguir nuestra propia inclinación natural.

Como la razón no es idónea para dirigir a la voluntad con miras al alcance de la felicidad; hemos de aceptar que la inclinación es más adecuada para llevarnos a tal propósito (vorPaGel da Silva, 2016). Resulta entonces que el fin propio de la razón es producir una voluntad buena en sí misma:

En efecto, como la razón no es bastante apta para dirigir de un modo seguro a la voluntad en lo que se refiere a los objetos de ésta y a la satisfacción de nuestras necesidades, pues a tal fin nos habría conducido mejor un instinto natural congénito, y sin embargo nos ha sido concedida la razón como facultad práctica, es decir, como una facultad que debe tener influjo sobre la voluntad, resulta que el destino verdadero de la razón tiene que ser el de producir una voluntad buena, no en tal o cual sentido, como medio, sino buena en sí misma. (Kant, 1985, p. 57)

Claro es que la voluntad puede estar motivada por la inclinación o por la razón: si el móvil de la voluntad es la inclinación, a lo sumo lo que lograríamos sería alcanzar la felicidad o cierto bienestar, mas la inclinación no está en capacidad de producir una voluntad buena, ya que la felicidad no tiene nada que ver con la moralidad. Si el móvil de la voluntad es la razón, ella no tiene la misión de lograr la felicidad - esto es propio de la inclinación-sino de producir una voluntad buena.

Kant ha afirmado enfáticamente que la voluntad es buena en sí misma (Rivadulla Durán, 2019); ahora nos va a decir cómo se produce una voluntad buena. Para desarrollar el tema de la voluntad apreciada en sí misma, hay que considerar el concepto de deber.

Al hablar de deber, el autor introduce una distinción fundamental: las acciones pueden hacerse conforme al deber, o por deber. Para explicar este asunto enumera una serie de ejemplos: la honradez del comerciante, el amor al prójimo, la búsqueda de la felicidad, el respeto por la 
propia vida, el cumplimiento de una promesa. Todas estas acciones se pueden realizar en vista al propio beneficio y no por deber (Aparicio-Gómez, 2019). Un comerciante que entregue el cambio correcto puede ser honrado, no a causa de que es su deber ser honesto, sino porque la honestidad trae buenos resultados al atraer a la clientela y aumentar la ganancia:

Por ejemplo, es conforme al deber, desde luego, que el comerciante no cobre más caro a un comprador inexperto, y en los sitios donde hay mucho comercio el comerciante avispado no lo hace, en efecto, sino que mantiene un precio fijo para todos en general, de forma que un niño pueda comprar en su tienda tan bien como otro cualquiera. (Kant, 1985, p. 59)

Dicho hombre es honrado porque con su actitud se atrae beneficios para sí mismo; de no obtener ningún beneficio, es probable que dicho individuo actuara de otra manera. En consecuencia, un individuo puede cumplir con su deber por razón de móviles egoístas o altruistas; si así actúa, su acción responde a la conformidad con el deber y no al puro deber, en otras palabras, actúa impulsado por la inclinación. En definitiva, una acción es conforme al deber cuando lo que se busca en el fondo es el beneficio personal.

Según Kant, una acción se hace por puro deber cuando en ella no influye la inclinación; si esto es así, dicha acción se puede decir que no tiene contenido moral. Este es el caso de un hombre que, no teniendo motivos para vivir, conserva su vida:

Cuando las adversidades y una pena sin consuelo han arrebatado a un hombre todo el gusto por la vida, si este infeliz, con ánimo fuerte y sintiendo más indignación que apocamiento o desaliento, y aun deseando la muerte, conserva su vida sin amarla sólo por deber y no por inclinación o miedo, entonces su máxima sí tiene un contenido moral. (Kant, 1985, p. 60)

La única acción que tiene contenido moral es la que se hace por deber; esta acción excluye todo influjo de la inclinación. Todo lo que se hace conforme al deber, pero siguiendo la 
inclinación, carece de contenido moral (Charpenel Elorduy, 2011). El problema de este tipo de hombres es que, si no los mueve la inclinación, entonces no actúan correctamente, no cumplen con su deber, en otras palabras, teniendo la posibilidad de hacer el bien, de cumplir con su deber, pueden en determinados casos no hacerlo.

Con este asunto del deber, la moral kantiana entra sin remedio a polemizar con las corrientes empiristas y utilitaristas de su tiempo:

Todos estos moralistas han mantenido que, cuando nos sentimos movidos a hacer algo únicamente por sentido del deber, no hemos alcanzado todavía la madurez moral; esta madurez se demuestra mucho mejor cuando no nos sentimos tentados a realizar el acto prohibido, cuando actuar justamente se ha convertido en parte de nuestra naturaleza hasta el punto de que ya ni siquiera tenemos que pensar en el deber. Si pudiéramos alcanzar este estado en todos los aspectos de nuestras vidas morales, los términos 'actuar por deber' desaparecerían de nuestro vocabulario moral. (Jospers, 1964, p. 390)

Por otra parte, el propósito con que se realice la acción no tiene nada que ver con el puro deber, pues no se trata de buenas intenciones; ya que cuando un sujeto realiza una acción en vista a un propósito, está motivado por algún tipo de placer que seguramente le traerá algún beneficio. ¿En qué consiste una acción realizada por puro deber? "El deber es la necesidad de una acción por respeto a la ley" (Kant, 1985, p. 63). Tal como lo entiende Kant, dicho respeto no se refiere a ninguna forma de sentimiento, sino a un modo especial como la razón concibe la obligatoriedad del deber. Es un respeto que no procede del sentimiento sino de la razón. En últimas, el valor moral de la acción no reside ni en la inclinación ni en el propósito que se espera, sino en el obrar por pura legalidad.

No queda, pues, otra cosa que pueda determinar a la voluntad, más que, objetivamente la ley, y subjetivamente el respeto puro a esa ley práctica y, por tanto, la máxima de obedecer siempre a esa ley, incluso en prejuicio de todas las inclinaciones. (Kant, 1985 , p. 64) 
La razón es entonces el fundamento determinante de la voluntad, en tanto que es ella la que formula la ley, sin cimentarse en ningún elemento empírico. Por tanto, el valor moral de la acción no puede residir en la relación de la voluntad con los efectos esperados.

Kant se pregunta ahora por el tipo de ley moral que debe servir de móvil a la voluntad para determinarla, para hacerla buena en sí misma, y responde a dicho interrogante con las siguientes palabras: "yo no debo obrar nunca más que de modo que pueda querer que mi máxima se convierta en ley universal" (Kant, 1985, p. 65). Esta ley expresa la mera legalidad; propiamente la autenticidad de dicha ley radica en el hecho de que se pueda universalizar. Si se me pregunta ¿por qué debo cumplir dicha ley? la respuesta es, porque debo cumplirla; esta es la forma de la mera legalidad. De esta manera ha llegado el filósofo de Königsberg a la formulación del imperativo categórico, aunque no le dé todavía ese nombre; esto lo explicará en el siguiente capítulo.

Esta ley nos sirve para determinar si la acción que se realiza es por deber o conforme al deber; todo depende de la posibilidad de universalización de dicha máxima, y de la intención con que se realice. Para ilustrar esto, Kant nos presenta el caso de las promesas: si prometemos algo con intención de no cumplirlo, sería como querer que nadie prometiese nunca nada, ya que, si intentamos universalizar esta acción, ella se destruiría a sí misma, pues de ante mano nadie confiaría en las promesas de nadie (Placencia, 2013). En últimas, sería como querer promesas sólo para el momento que nos convenga; el resultado de esto es que algo así no tendría sentido; aparte de que nadie nos creería, nos estaríamos contradiciendo. Precisamente el criterio de la universalización nos mostraría dicha contradicción.

Para llevar a cabo este análisis basta sólo con preguntar lo siguiente: ¿puedo querer que mi máxima se convierta en ley universal? Si la universalización de la máxima implica su autodestrucción, entonces tal máxima no puede ser moral (Aparicio-Gómez, 2016). ¿Puedo querer que todos los que hacen promesas las rompan? En ese caso nadie creería en las promesas y éstas dejarían de existir. 
Por otra parte, nos podríamos mantener en pie con la promesa y efectivamente cumplir con el deber, pero no por puro deber, sino que nos movamos a hacerlo por miedo o por la ganancia que nos reporta. Este es un asunto que Kant tratará mas adelante; veremos que el tratar de determinar en concreto si una acción se realiza conforme al deber o por deber resulta en la práctica imposible: "Es imposible determinar por la experiencia si una acción ha sido realizada por puro deber o es conforme al deber" (Kant, 1985, p. 72). Esto sólo lo puede hacer el sujeto que realiza la acción. Como dice el mismo Kant, "cuando se trata del valor moral no importan las acciones que se ven, sino sus principios íntimos, que no se ven” (Kant, 1985, p. 72).

Finalmente, en este capítulo Kant se refiere a la necesidad de pasar del conocimiento común al filosófico. Esto por razón de ir escalando en el análisis con el objetivo de descubrir el lugar donde se asientan u originan los conceptos básicos de la moral y, en consecuencia, la posible ley que nos pueda servir como herramienta de enjuiciamiento de nuestras acciones morales. Según Kant, si nos dedicamos a buscar los fundamentos en la inclinación, nuestras acciones se realizarían básicamente en conformidad con el deber y no por deber (Norbert, 1992). En consecuencia, no podríamos hablar de valor moral, ni de una ley objetiva. Pues nuestra naturaleza humana ordinaria está expuesta a multitud de necesidades y estímulos distintos que, en la mayoría de los casos, van en contra del deber. De ahí que una buena voluntad sea la que realiza su acción única y exclusivamente por amor al puro deber y no por inclinación.

\section{Conclusiones}

. La buena voluntad no es buena por lo que realice, o por el fin que se pretenda alcanzar.

. Todos los hombres aspiran a la felicidad.

. La voluntad es buena en sí misma.

. La única acción que tiene contenido moral es la que se hace por deber.

. Si nos dedicamos a buscar los fundamentos en la inclinación, nuestras acciones se realizarían básicamente en conformidad con el deber y no por deber. 


\section{Referencias}

Anzenbacher, Arno (1993). Introducción a la filosofía. Madrid. Herder.

Aparicio-Gómez, Oscar-Yecid (2006). El nexo familia persona en la filosofía personalista

(Tesis Doctoral). Barcelona, España: Universidad de Barcelona. Disponible en: http://hdl.handle.net/2445/41696

Aparicio-Gómez, Oscar-Yecid (2016). La persona en la Sociedad Digital. En AparicioGómez, Oscar-Yecid (Eds.). El Uso Educativo de las TIC (pp. 251-256). Bogotá, Colombia: Universidad Central. ISBN: 978-958-26-0316-8

Aparicio-Gómez, William-Oswaldo (2019). The community dynamics as a divine pedagogy. En: Revista Interamericana de Investigación, Educación y Pedagogía, RIIEP. Vol. 12, Núm. 1. p. 55 - 71. ISSN: 1657-107X - e-ISSN: 2500-5421. DOI: 10.15332/s1657107X.2019.0001.08

Avila Martinez, A. (2014). La filosofía del derecho de I. Kant. Una perspectiva desde la crítica de la razón práctica y Metafísica de las costumbres. Criterio Jurídico Garantista. https://doi.org/10.26564/21453381.5

Carvalho, Z. de J. V. de. (2015). Considerações sobre a aparência a partir de rousseau e kant: aproximações e diferenças. Cadernos de Pesquisa. https://doi.org/10.18764/cp.v22i0.2977

Charpenel Elorduy, E. Ó. (2011). Los sentimientos y el deber en la filosofía práctica de Kant. Open Insight. https://doi.org/10.23924/oi.v2n2a2011.pp5-29.22

Colomer, E. (2006). El pensamiento alemán de Kant a Heidegger. Tomo I. La filosofía trascendental: Kant. Madrid. Herder. 
Del Luján Di Sanza, S. (2014). Kant, Immanuel, Fundamentación para una metafísica de las costumbres. Versión castellana y estudio preliminar por Roberto R. Aramayo. Alianza Editorial. "El libro de bolsillo", Madrid. Segunda Edición, 2012. Con-Textos Kantianos. International Journal of Philosophy. https://doi.org/10.5281/zenodo.18455

Gallo, H. (2015). Fundamentos de la ética TT - Fundamentals of ethics. Affectio Soc. (Medellín).

González, L. (2019). Parra, Lisímaco. "Filosofía versus barroco en la Fundamentación de la metafísica de las costumbres de Kant”. Ideas y Valores. https://doi.org/10.15446/ideasyvalores.v68n170.77706

Iracheta Fernández, F. (2009). El problema de la libertad y de la normatividad en la teoría ética de Kant. Dikaiosyne.

Jospers, John. (1964). La conducta Humana. Madrid. Tecnos.

Kant, Immanuel (1985). Fundamentación de la metafísica de las costumbres. Madrid. EspasaCalpe.

Lafarga, F., \& Pegenaute, L. (2016). Fundamentación de la metafísica de las costumbres de I. Kant, en traducción de Manuel García Morente (1921) 407. In Cincuenta estudios sobre traducciones españolas. https://doi.org/10.3726/978-3-0351-0188-1/47

Martínez Martínez, J. P. (2017). La interpretación del mandamiento del amor en la ética kantiana. The Interpretation of Love Commandment in Kantian Ethics. Metafísica y Persona. https://doi.org/10.24310/metyper.2015.v0i13.2723

Norbert, B. (1992). ¿Cómo Pensar los Valores Morales a Partir de Kant? Anales Del Seminario de Historia de La Filosofía.

Placencia, L. (2013). Kant y la voluntad como "razón práctica". kant and the will as practical reason. Tópicos, Revista de Filosofía. https://doi.org/10.21555/top.v41i1.75 
Plata Pineda, O. (2011). El yo, el otro-yo y el otro en Kant. de la fundamentación de la metafísica de las costumbres a la religión dentro de los límites de la razón. Praxis Filosófica. https://doi.org/10.25100/pfilosofica.v0i19.3220

Rivadulla Durán, A. (2019). El concepto de amistad en Kant. Isegoría. https://doi.org/10.3989/isegoria.2019.061.03

VorPaGel da Silva, L. (2016). Sobre el problema de la libertad en On the problem of freedom in Kant. 
REVISTA INTERNACIONAL DE FILOSOFÍA TEÓRICA Y PRÁCTICA

Volumen 1. Número 2. Julio - Diciembre 2021 ISSN: 2745-0333 (En línea) 